\title{
Multicellular Spheroids as a Model for Hypoxia-induced EMT
}

\author{
CASEY LEE, AMANDA SIU and DANIEL M. RAMOS \\ Department of Orofacial Sciences, University of California at San Francisco, San Francisco, CA, U.S.A.
}

\begin{abstract}
Oral cancer is aggressive and invasive. The 5-year survival rate is around $50 \%$ and has not improved in over 50 years. One-third of oral cancer patients develop local and/or regional tumor recurrence following treatment. We continue to use our multicellular spheroid (MCS) model to better understand how the extracellular matrix contributes to epithelial to mesenchymal transition and how hypoxia contributes to the progression of oral squamous cell carcinoma (SCC).
\end{abstract}

Oral cancer is the sixth most common type of cancer in the world. In the United States, 37,000 new cases are diagnosed annually and each year 8,000 individuals die from this disease (1). Squamous cell carcinoma (SCC) makes up 96\% of all oral cancers (2). Despite advances in treatment, the prognosis for oral SCC has not improved in over 50 years. The 5-year survival rate for oral SCC is approximately $50 \%$ (3). The poor prognosis of SCC is due to invasion and local recurrence. Interactions between primary SCC cells and the surrounding extracellular matrix (ECM) facilitate invasion. As a tumor grows its available resources, including oxygen availability, become extremely limited which may affect tumor cell behavior. In this study, we aimed to determine the effect of hypoxia on EMT in OSCC spheroids.

SCC cells undergo a transformative process known as epithelial-mesenchymal transition (EMT). EMT is a process by which epithelial cells lose their cell polarity, decrease cellcell adhesion, and gain migratory and invasive properties. EMT involves changes in expression, distribution, and function of a number of proteins for cells to transition between the epithelial and mesenchymal phenotype. During EMT, Ecadherin expression is lost; thereby disrupting desmosomes,

This article is freely accessible online.

Correspondence to: Daniel M. Ramos, DDS, Ph.D., Department of Orofacial Sciences, University of California, 513 Parnassus Ave, S612, Box 0422, San Francisco, CA 94143-0422, U.S.A. Tel: +1 4155024905, Fax: +1 8007836653, e-mail: Daniel.ramos@ucsf.edu

Key Words: Spheroid, multicellular spheroid formation, EMT, Hypoxia. promoting invasion and de-differentiation (4). N-cadherin acquisition also promotes invasive $\mathrm{SCC}$ behavior $(4,5)$.

Previous studies show that the $\alpha v \beta 6$ integrin, an epithelial specific integrin, is instrumental to EMT in colorectal and oral cancer $(6,7)$. The $\alpha v \beta 6$ integrin is an epithelial-specific adhesion receptor normally not expressed in oral keratinocytes. However, upon wounding or neoplastic transformation, $\alpha v \beta 6$ is highly expressed by the same keratinocytes. The $\alpha v \beta 6$ integrin is an adhesion receptor for fibronectin (FN) and tenascin-C, and also activates TGF $\beta 1(8,9)$. Binding of $\alpha v \beta 6$ integrin to FN results in the activation of FYN kinase in oral SCC. The activation of FYN kinase promotes activation of RAF/MAPK which induces EMT (10). Our laboratory demonstrated that expression of the full-length $\beta 6$ subunit is required for maintenance of the mesenchymal phenotype thus promoting EMT (7).

Keratins are the major structural components of the epithelial cell cytoskeleton and play a strategic role in their architectural integrity (11). It has been shown that gene mutations in keratin are responsible for a large number of inherited skin disorders thus, reinforcing their role in structural integrity of the epithelium (11). In addition to cytoskeletal changes, it is a common process for the ECM to undergo a variety of changes during tumor progression. For example, as breast tissue undergoes dysplastic changes there is a shift in the deposition of FN, which induces EMT (12).

Tumor hypoxia is strongly associated with tumor growth and malignant progression. Hypoxia is a non-physiological level of oxygen tension, a common phenomenon in a majority of malignant tumors (13). Tumor-hypoxia can alter vascularization and help the acquisition of epithelial-to-mesenchymal transition phenotype, which can result in enhanced cell mobility and metastasis (15). Hypoxia in the tumor microenvironment may also play a role in conferring resistance to therapy and it is currently a focus of cancer treatment since many solid tumors display an additional growth advantage through their ability to survive in a hypoxic environment (14). HIF1 $\alpha$ is up-regulated by oxygen deprivation and is associated with increased patient mortality in several cancer types (15). As a tumor grows its available resources, including oxygen availability, become extremely limited which may affect tumor cell behavior. In this study, we aimed to determine the effect of hypoxia on EMT in OSCC spheroids. 


\section{Materials and Methods}

Cell lines. The SCC9 cell line was derived from a base of tongue cancer and was a generous gift from Dr. James Reinwald (Brigham and Woman's Hospital, Harvard School of Medicine). The SCC9 $\beta 6$ cell line was established by stable transfection of SCC9 cells with full length $\beta 6$ cDNA (17). SCC9 $\beta 6$ cells were transduced with the kinase-dead (KD) Fyn cDNA in order to establish the SCC9ß6KDFyn cell line using the Retro-X system (Clontech, Mountain View, CA, USA) $(16,17)$. The SCC9 cells were transduced with constitutively active (CA) Fyn cDNA to establish the SCC9CAFyn cells using the Retro-X system (Clontech, Mountain View, CA, USA) (10). The KDFyn cDNA was a generous gift from $\mathrm{Dr}$ H. Kawakatsu (University of California, San Francisco). The cDNA for the full-length $\beta 6$ was a generous gift from Dr. Dean Sheppard (University of California, San Francisco).

Generation of Multicellular Spheroids. Tissue culture plates (Santa Cruz Biotechnology, Dallas, TX, USA) were treated with a $0.6 \%$ agarose solution and air-dried overnight at room temperature. Cells were harvested from monolayer cultures with $0.25 \%$ trypsin-EDTA and rinsed twice to remove serum. The cells were then placed on agarose-coated plates and allowed to form multicellular spheroids for $48 \mathrm{~h}$ at $37^{\circ} \mathrm{C}$ in $5 \% \mathrm{CO}_{2}$ in serum free media.

Antibodies. Rabbit monoclonal antibodies to vimentin (clone R28) (CST\#3932) (1:1000 dilution), HIF1 $\alpha$ (CST \#3716) (1:1,000 dilution), and $\beta$-actin (clone 13E5) (CST\#4970) (1:1,000 dilution) were purchased from Cell Signaling Technology Inc.(Danvers, MA, USA). Mouse monoclonal antibody to pan-keratin (clone C11) (CST \#4545) (1:1,000 dilution) was purchased from Cell Signaling Technology Inc., (Danvers, MA, USA). Mouse monoclonal antibody to fibronectin (clone FN-15) (1:800 dilution) was purchased from Sigma Aldrich (St. Louis, MO, USA). Horseradish peroxidase (HRP)-conjugated anti-rabbit antibodies (CST\#7074) (1:2,000 dilution) and HRP-conjugated anti-mouse antibodies (CST \#7076) (1:2,000 dilution) were purchased from Cell Signaling Technology Inc. Cobalt (II) chloride hexahydrate (C8661) (100 $\mu \mathrm{M})$ was purchased from Sigma Aldrich (St. Louis, MO, USA).

Western blot. Cells were grown as a monolayer or as a multicellular spheroid (MCS) in serum-free media for $48 \mathrm{~h}$ prior to lysing. Cells were rinsed with ice-cold $1 \mathrm{X}$ PBS and lysed. Total protein was extracted from cells with RIPA lysis buffer $(50 \mathrm{mM}$ Tris $\mathrm{pH} 8$, $150 \mathrm{mM} \mathrm{NaCl}, 0.2 \%$ SDS, $1 \%$ deoxycholic acid, $1 \%$ Triton X-100, and complete protease inhibitor tablet). Lysate was generated by suspending MCS in RIPA lysis buffer, vortexing, and then forcing MCS suspension through a 30-gauge needle. Monolayer lysate was generated by pipetting RIPA lysis buffer over the culture and scraped with a cell scraper. Twenty $\mu \mathrm{g}$ of protein per cell line was resolved by SDS-PAGE and transferred to a PVDF membrane using a semi-dry apparatus (Bio-Rad, Hercules, CA, USA). Membranes were incubated with monoclonal antibodies overnight at $4^{\circ} \mathrm{C}$. After washing, the blots were incubated with HRP-conjugated anti-rabbit antibodies for 40 minutes. The blots were then treated with ECL substrate (Thermo Scientific Pierce; Rockford, IL, USA) and exposed to x-ray film (Santa Cruz Biotechnology). Western Blot images were quantified using NIH Image J (Image J reference) (18). $\beta$-actin or tubulin were used as control bands to quantify the image. The images were digitally captured and assigned relative value units based on the intensity of the signal. To establish relative value units, the ImageJ was used toolbar to draw a rectangle around the control lane (tubulin or actin). This is repeated for each peak. Each peak with its size, is expressed as a percentage of the total size of all of the highlighted peaks. Each subsequent percentage is then divided by the percentage of the assigned control.

\section{Results}

Expression of keratin is differentially expressed by cells lines and multicellular spheroids. To evaluate keratin expression, the oral SCC cell lines were grown serum-free, either as a monolayer or MCS for $48 \mathrm{~h}$. The cells were lysed, normalized, and evaluated for expression of keratin by western blot analysis. The blots were scanned, digitized and assigned a relative value unit (rvu).

When grown as a monolayer and processed for western blot analysis, SCC9 cells were assigned an rvu of 1.0 (Figure 1). This was in contrast to the SCC $9 \beta 6$ and the SCC9CAFyn cell lines, which were assigned relative value units of 2.0 (Figure 1). Keratin was undetectable in the SCC9 36 KDFyn cell line. However, when cells were grown as multicellular spheroids, keratin expression was up-regulated by all cell lines compared to their corresponding monolayers. SCC9, SCC9CAFyn, and SCC9 $\beta 6$ cells expressed keratin with similar rvu(s) $(3.9,3.7$ and 3.3, respectively) (Figure 1). The SCC9 $36 \mathrm{KDFyn}$ cell line did not express any detectable keratin when grown as a monolayer but when grown as a MCS, it expressed low level keratin (rvu: 0.7) (Figure 1), suggesting a neo-expression of keratin in SCC9 36 KDFyn MCS. Our results suggest that the three-dimensional cell-cell contact, which occurs during MCS growth, modulates expression of keratin.

MCS formation results in impaired expression of vimentin. Vimentin (VIM) is an intermediate filament routinely found in mesenchymal cells and used to detect cells progressing through EMT (19). When the oral SCC cells were grown as a monolayer, the SCC9CAFyn and SCC9 36 KDFyn cell lines expressed VIM with an rvu of 1.0 and 3.5 ; respectively (Figure 2). Trace amounts of VIM were detected in the SCC9 and SCC9 $\beta 6$ cell lines each with an rvu of 0.2 and 0.1 , respectively (Figure 2). This modest expression of VIM was reduced even more when the cells were grown as an MCS, where VIM was detected only in the SCC9 36 KDFyn cell line with an rvu of 2.5 (Figure 2).

Fibronectin is differentially expressed by the SCC cells both in monolayers as well as in a MCS. Fibronectin (FN) is a key molecule of ECM and has been identified as a marker for EMT. As a glycoprotein dimer, FN is composed of similar subunits (220 kDa and $240 \mathrm{kDa}$ ) linked by 2 disulfide bonds at their C-terminus. To investigate FN's expression, SCC cells were first grown as a monolayer and then evaluated by western blot analysis. A robust expression of FN was detected 


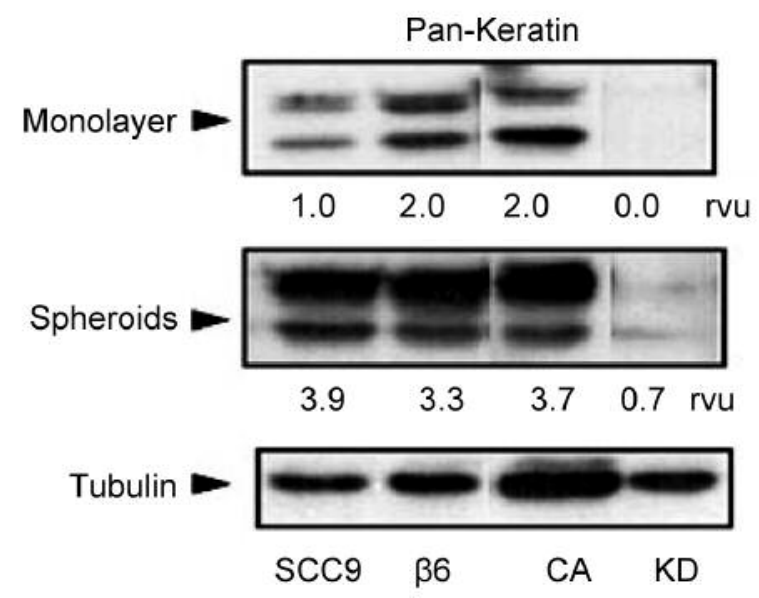

Figure 1. Differential expression of keratin in oral SCC monolayers $v s$. multicellular spheroids (MCS). SCC9, SCC9 $\beta 6$ ( $\beta 6)$ ), SCC9CAFyn (CA) and SCC9ß6KDFyn (KD) cells were grown for $48 h$ either as a monolayer or MCS under serum-free conditions and analyzed by western blotting using a pan-keratin Ab. Expression of keratin was increased in all cell lines when grown as an MCS.

in SCC9 and SCC9 36 cells (rvu: 2.8 and 2.5; respectively) (Figure 3) while SCC9 $36 \mathrm{KDFyn}$ and SCC9 $36 \mathrm{CAFyn}$ cells expressed less FN and had an rvu of 1.0 and 0.8 , respectively (Figure 3).

Three-dimensional growth was next evaluated by growing the cells as an MCS. With the exception of the SCC9 36 KDFyn cells, overall FN expression of SCC9, SCC9 $\beta 6$ and SCC9CAFyn cells was decreased (rvu: of 1.0,2.2, and 0.3) (Figure 3). Surprisingly the expression of $\mathrm{FN}$ by the SCC936KDFyn cell line increased $400 \%$ and was assigned an rvu of 4.3 (Figure 3). These results demonstrate a differential expression of FN by the oral SCC cells when grown in a three dimensional environment. These results, plus our previous work demonstrating that other EMT markers ( $\mathrm{N}$-cadherin and c-Raf) were upregulated in SCC9 96 KDFyn MCS, indicate that the SCC9 $36 \mathrm{KDFyn}$ cell line is most poised to enter into EMT $(7,20,21)$.

Hypoxia and tumor MCS. SCC9 $96 \mathrm{KDFyn}$ cells are the most potentiated to initiate $\operatorname{EMT}(7,14)$. Due to the close relationship between tumor hypoxia and EMT, we used the SCC9 36KDFyn cells to study hypoxia $(7,20,21)$. The SCC9 36 KDFyn cells express $\mathrm{N}$-cadherin rather than Ecadherin when grown as an MCS, which is indicative of a motile phenotype (21). To simulate hypoxia, the cultures were incubated in the presence of $\mathrm{CoCl}_{2}$ for $48 \mathrm{~h}$. SCC9 $36 \mathrm{KDFYN}$ cells grown as MCS under normoxic condition expressed FN (rvu:3.0) (Figure 4). However, when grown in the presence of $\mathrm{CoCl}_{2}, \mathrm{FN}$ expression was suppressed by $60 \%$ (rvu:1.2)



Figure 2. Mutations to Fyn kinase modulate the expression of vimentin. The SCC9, SCC9 66 ( $\beta 6$ ), SCC9CAFyn (CA) and SCC9 66 KDFyn (KD) cells were grown either as a monolayer or an MCS and then examined for the expression of vimentin. When grown as a monolayer trace amounts were detected in the SCC9 and the SCC9 66 cells and more substantial levels were detected in the SCC9CAFyn and SCC9 66 KDFyn cells. However when grown as an MCS, vimentin was detected only in the SCC9B6KDFyn cell line.

(Figure 4). SCC9 66 KDFyn cells were also analyzed for $\mathrm{N}$-cadherin expression. $\mathrm{N}$-cadherin expression decreased by $60 \%$ when treated with $\mathrm{CoCl}_{2}$. When SCC9 $36 \mathrm{KDFyn}$ cells were grown as an MCS in a normoxic environment, HIF1 $\alpha$ was undetectable. However, when the same cells were incubated in the presence of $\mathrm{CoCl}_{2}, \mathrm{HIF} 1 \alpha$ was found to be neo-expressed (rvu: 1.0) (Figure 4). These results indicate the SCC9 36KDFyn cells are able to sustain themselves even in a low oxygen environment and this may confer a growth advantage.

\section{Discussion}

The protein content of various oral SCC cells can be indicative of the natural history of the disease. Keratins are the intermediate filament-forming proteins of epithelial cells. The filaments have an important role in epithelial cell protection from mechanical or non-mechanical stresses (22). More recently, keratins have also been recognized as regulators of other cellular properties and functions, including apico-basal polarization, motility, cell size, protein synthesis, and membrane trafficking and signaling. Keratin is altered by malignant transformation and can be used to identify tumors of epithelial origin (23). In this study, we evaluated how oral SCC cells modulate the expression of keratin when grown as a three-dimensional structure composed entirely of tumor cells. When grown in a suspended three-dimensional MCS, all SCC cell lines upregulated keratin expression. SCC9 36 KDFYN cells, which did not express keratin when was grown as a 


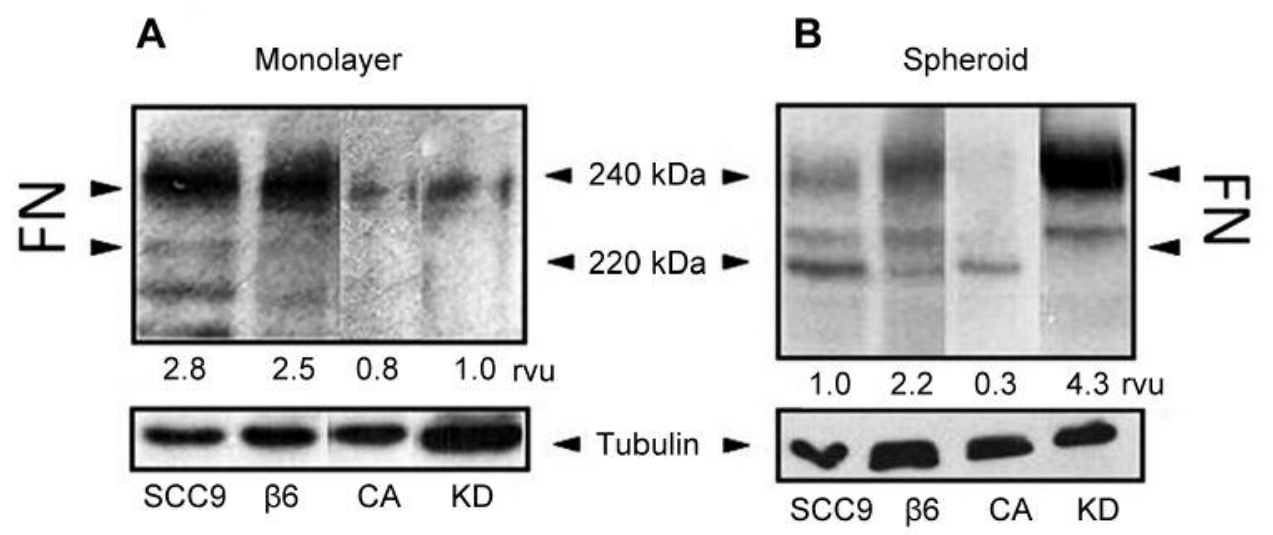

Figure 3. Fibronectin expression is altered by multicellular spheroid formation. (A) SCC9, SCC9 36 ( $\beta 6$ ), SCC9CAFyn (CA) and SCC9 $66 K D F y n$ (KD) cells were grown as a monolayer for $48 \mathrm{~h}$ and evaluated for fibronectin. SCC9 and the SCC9 36 cells expressed more FN (rvu: $2.8,2.5$ respectively) compared to SCC9CAFyn and SCC9 $66 \mathrm{KDFyn}$ cells (rvu: 0.8, 1.0 respectively). (B) When cells were grown as an MCS for $48 \mathrm{~h}$ and evaluated for fibronectin, the overall expression of FN was decreased with the exception of the SCC9B6KDFyn cell line which showed an rvu of 4.
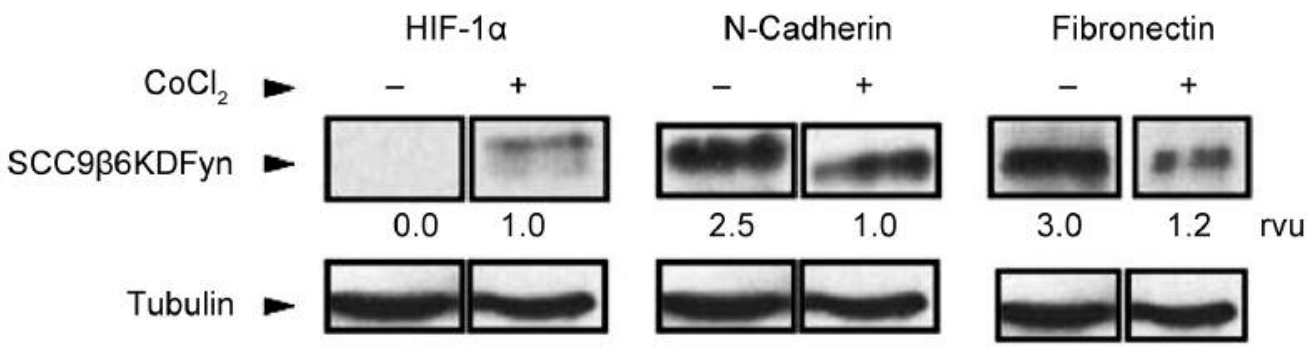

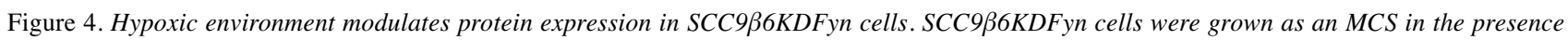
or absence of $\mathrm{CoCl}_{2}$ for $48 \mathrm{~h}$ at $37^{\circ} \mathrm{C}$ to induce a hypoxic environment. $\mathrm{HIF}$-1 $\alpha$ expression was (rvu: 1.0); whereas expression of $N$-cadherin and FN were decreased approximately $66 \%$ for both (rvu 1.0 and 1.2; respectively).

monolayer, expressed keratin when grown in MCS (Figure 1).

VIM, a major constituent of the intermediate filament family of proteins, is ubiquitously expressed in normal mesenchymal cells and like keratin, is also a structural protein that helps maintain cell structure and resistance to external stresses. Loss of E-cadherin coupled with $\mathrm{N}$ cadherin expression and acquisition of a fibroblast-like morphology are contributing factors to the process known as epithelial-mesenchymal-transition (EMT). In recent years, VIM has been recognized as a marker for $\operatorname{EMT~}(7,21)$. VIM is overexpressed in various epithelial cancers, including prostate, gastrointestinal, central nervous system, breast, melanoma and lung cancer. Overexpression of VIM in cancer correlates well with accelerated tumor growth, invasion and poor prognosis (24). When SCC cells were grown as a monolayer, those containing the Fyn Kinase mutations (kinase-dead or a constitutively active) expressed elevated VIM, indicating an association between Fyn kinase stability and the expression of VIM (Figure 2). When grown as an MCS only the SCC9 $36 \mathrm{KDFYN}$ cells (kinase-dead FYN) expressed VIM but even this expression was reduced by $33 \%$ compared to cells grown in monolayer (Figure 2).

Hypoxia alters cancer cell metabolism and contributes to therapy resistance by inducing cell quiescence. Hypoxia stimulates complex cell signaling networks in cancer, including HIF, PI3K, MAPK, and NFkB pathways which interact with each other causing positive and negative feedback loops resulting in enhanced or diminished hypoxic effects (25). Over expression of HIF1 $\alpha$ may play an adverse role in the malignant progression of head and neck cancer by facilitating the adaptation of cells to the hypoxic state; which then contributes to the invasive properties and angiogenesis in these tumors. Hypoxia is a common characteristic of solid tumors where the centers are typically necrotic (26). Cells that can survive in the absence of oxygen are therefore at a growth advantage compared to cells that cannot. Therefore, MCS, which mimic 
in vivo tumors with proliferative cells in the periphery and hypoxic centers (27), were used to evaluate hypoxia in vitro. When SCC9 36 KDFyn cells were placed under hypoxic conditions a variety of changes occurred such as a 3-fold decrease in $\mathrm{FN}$ expression and a 2.5 -fold decrease in $\mathrm{N}$ cadherin expression (Figure 4). Tumor growth is complex and our understanding of the process continues to grow. Tumor cells are sensitive to their immediate environment and one might think of that as far targeted therapy. Expression and secretion of specific matrix proteins alters the immediate extracellular matrix. Our results indicate that cellular growth as MCS alters oral SCC cells' protein expression profile and may represent a more realistic cellular model in relation to what is taking place in vivo.

\section{References}

1 Oral Cancer Fact Sheet. NIH, 2010.

2 Messadi DV: Diagnostic aids for detection of oral precancerous conditions. Int J Oral Sci 5: 59-65, 2013.

3 Howlader N, Noone AM, Krapcho M, Miller D, Bishop K, Altekruse SF, Kosary CL, Yu M, Ruhl J, Tatalovich Z, Mariotto A, Lewis DR, Chen HS, Feuer EJ and Cronin KA (eds): SEER Cancer Statistics Review, 1975-2013, National Cancer Institute. Bethesda, MD, http://seer.cancer.gov/csr/1975_2013/ page 34, based on November 2015 SEER data submission, posted to the SEER web site, April 2016.

4 Beck TN, Chikwem AJ, Solanki NR and Golemis EA: Bioinformatic approaches to augment study of epithelial-tomesenchymal transition in lung cancer. Physiol Genomics 46(19): 699-724, 2014.

5 Umbreit C, Erben P, Faber A, Hofheinz RD, Schultz JD, Hoermann K and Wenzel A: Lapatinib-induced mesenchymalepithelial transition in squamous cell carcinoma cells correlates with unexpected alteration of $\beta$-catenin expression. Oncol Lett 11(4): 2715-2724, 2016.

6 Bates RC: Colorectal Cancer Progression: integrin alphavbeta6 and the epithelial-mesenchymal transition (EMT). Cell Cycle 4: 1350-1352, 2005.

7 Lee C, Lee C, Atakilit A, Siu A and Ramos DM: Differential Spheroid formation by Oral Cancer Cells. Anticancer Res 34(12): 6945-6949, 2014.

8 Dang D, Yang Y, Li X, Atakilit A, Regezi J, Eisele D, Ellis D and Ramos DM: Matrix Metalloproteinases and TGF $\beta 1$ modulate oral tumor cell matrix. Biochem Boiphys Res Commun 316: 937-942, 2004.

9 Munger JS, Huang X, Kawakatsu H, Griffiths MJ, Dalton SL, Wu J, Pittet JF, Kaminski N, Garat C, Matthay MA, Rifkin DB and Sheppard D: The integrin alpha $\mathrm{v}$ beta 6 binds and activates latent TGF beta 1: a mechanism for regulating pulmonary inflammation and fibrosis. Cell 96: 319-328, 1999.

10 Lewin B, Siu A, Baker C, Dang D, Schnitt R, Eisapooran P and Ramos DM: Expression of Fyn Kinase Modulates EMT in Oral Cancer Cells. Anticancer Res 30(7): 2591-2596, 2010.

11 Yoon KH, Yoon M, Moir RD, Khuon S, Flitney FW and Goldman RD: Insights into the dynamic properties of keratin intermediate filaments in living epithelial cells. J Cell Biol 153(3): 503-516, 2001
12 Park J and Schwarzbauer JE: Mammary epithelial cell interactions with fibronectin stimulate epithelial-mesenchymal transition. Oncogene 33(13): 1649-1657, 2014.

13 Kao SH, Wu KJ and Lee WH: Hypoxia, Epithelial-Mesenchymal Transition, and TET-Mediated Epigenetic Changes. J Clin Med 5(2): 24, 2016.

14 DeBerardinis RJ and Chandel NV: Fundamentals of Cancer Metabolism. Oncology Sci Adv 2: e16002, 2016.

15 Semenza GL: Targeting HIF-1 for cancer therapy. Nat Rev Cancer 3(10): 721-732, 2003.

16 Ramos DM, Dang D and Sadler S: The role of the integrin alpha $\mathrm{v}$ beta6 in regulating the epithelial to mesenchymal transition in oral cancer. Anticancer Res 29: 125-130, 2009.

17 Li X, Yang Y, Hu Y, Dang D, Regezi J, Schmidt BL, Atakilit A, Chen B, Ellis D and Ramos DM: Alphavbeta6-Fyn signaling promotes oral cancer progression. J Biol Chem 278: 4164641653, 2003.

18 Rasband WS: Image J v1.6, U. S. National Institutes of Health, Bethesda, Maryland, USA, http://imagej.nih.gov/ij/, 1997-2015.

19 Zeisberg $M$ and Neilson EG: Biomarkers for epithelialmesenchymal transitions. J Clin Invest 119(6): 1429-1437, 2009.

20 Lee C and Ramos DM: Regulation of Multicellular Spheroids by MAPK and FYN Kinase. Anticancer Res 36: 3833-3838, 2016.

21 Lee C, Lee C, Lee S, Siu A and Ramos DM: The cytoplasmic extension of the integrin $\beta 6$ subunit regulates epithelial-tomesenchymal transition. Anticancer Res 34(2): 659-664, 2014.

22 Coulombe PA and Omary MB: 'Hard' and 'soft' principles defining the structure, function and regulation of keratin intermediate filaments. Curr Opin Cell Biol 14(1): 110-122, 2002.

23 Ray JG, Ranganathan K and Chattopadhyay A: Malignant transformation of oral submucous fibrosis: overview of histopathological aspects. Oral Surg Oral Med Oral Pathol Oral Radiol 122(2): 200-209, 2016.

24 Ivaska J, Pallari HM, Nevo J and Eriksson JE: Novel functions of vimentin in cell adhesion, migration, and signaling. Exp Cell Res 313(10): 2050-2062, 2007.

25 Muz B, De la Puente P, Azab F, Ghobrial IM and Azab AK: Hypoxia Promotes Dissemination and Colonization in New Bone Marrow Niches in Waldenström Macroglobulinemia. Mol Cancer Res 13(2): 263-272, 2014.

26 Tian X, Wang W, Zhang Q, Zhao L, Wei J, Xing H, Song Y, Wang S, Ma D, Meng L and Chen G: Hypoxia-inducible factor$1 \alpha$ enhances the malignant phenotype of the multicellular spheroid HeLa cells in vitro. Oncol Lett 1: 893-897, 2010.

27 Desmaison A, Frongia C, Grenier K, Ducommun B and Lobjois $\mathrm{V}$ : Mechanical stress impairs mitosis progression in multicellular tumor spheroids. PLoS One 8(12): e80447, 2013.
Received October 1, 2016

Revised October 17, 2016

Accepted October 24, 2016 